\title{
A 79-year-old man with unexplained recurrent syncope and severe orthostatic hypotension
}

\author{
Beatrice Laura Montinaro ${ }^{1,2} \cdot$ Viviana Bozzano ${ }^{1,3}$. Angelica Carandina ${ }^{1}$. Giorgio Alberto Croci ${ }^{4,5}$. \\ Alessio Di Fonzo ${ }^{6,7}$. Eleonora Tobaldini ${ }^{1,2}$ (])
}

Received: 25 May 2021 / Accepted: 17 August 2021 / Published online: 15 September 2021

(c) Società Italiana di Medicina Interna (SIMI) 2021

\section{Presentation of case}

Dr. Montinaro: a 79-year-old man was admitted to our hospital, because of diarrhoea and pre-renal acute kidney injury AKI. The gastrointestinal syndrome developed 3 days before the admission. The symptoms were not responsive to common antimotility agents, and the patient developed severe fatigue and syncopal prodromes in orthostatism.

The patient's medical history included paucisymptomatic idiopathic pulmonary fibrosis IPF with usual interstitial pneumonia pattern treated with protein-kinase inhibitor Nintedanib, chronic kidney disease, bilateral nephrolithiasis, benign prostatic hyperplasia and previous cholecystectomy.

His medical record was also notable for a 2-year history of relapsing episodes of transient loss of consciousness (T-LOC) that did not have specific triggers, such as defecation, cough or sneeze, post-exercise, or emotional triggers. Some episodes lead to falls with severe traumatic outcomes, and in one case, the patient had been hospitalized

Eleonora Tobaldini

eleonora.tobaldini@unimi.it

1 Division of Internal Medicine, Foundation IRCCS Ca' Granda Ospedale Maggiore Policlinico, 20122 Milan, Italy

2 Department of Clinical Sciences and Community Health, University of Milan, Milan, Italy

3 Division of Emergency Medicine, Foundation IRCCS Ca' Granda Ospedale Maggiore Policlinico, 20122 Milan, Italy

4 Division of Pathology, Foundation IRCCS Ca' Granda Ospedale Maggiore Policlinico, 20122 Milan, Italy

5 Department of Pathophysiology and Transplantation, University of Milan, Milan, Italy

6 Division of Neurology, Foundation IRCCS Ca' Granda Ospedale Maggiore Policlinico, Milan, Italy

7 Dino Ferrari Centre, Neuroscience Section, Department of Pathophysiology and Transplantation, University of Milan, Milan, Italy for post-traumatic subarachnoid haemorrhage and the c5 vertebrae fracture. At that time, sick sinus syndrome was identified, and the patient was implanted with a bicameral pacemaker (DDDR) and discharged with calcium channel blockers. However, runs of sustained atrial tachycardia were seen, and the patient was started on low-dose beta-blockers and anticoagulation with a direct oral anticoagulant. Despite the listed interventions, the patient kept on experiencing severe traumatic falls due to syncopal T-LOC, but pacemaker interrogation did not reveal any further rhythm anomalies.

On arrival at the emergency department, the patient was unable to keep the upright position because of asthenia. The axillary temperature was $36{ }^{\circ} \mathrm{C}$, the heart rate (HR) 78 beats per minute (bpm), the blood pressure (BP) in clinostatism was $120 / 90 \mathrm{~mm} \mathrm{Hg}$, and the oxygen saturation $98 \%$ while breathing in ambient air. The lungs presented bilateral crackles at the middle and lower fields, which were fibrosis-related findings previously described at pneumological follow-up. The heart was normal on auscultation, with rhythmic heart sounds and without detectable heart murmurs. He had diffused abdominal discomfort with point tenderness on palpation of the left flank, without signs of peritonism. Peripheral pulses were valid and symmetrical, and the skin was normally perfused.

Electrocardiography (ECG) showed sinus rhythm at $78 \mathrm{bpm}$, left anterior fascicular block, and some premature ventricular contractions, and was comparable to previous ECGs. On blood testing, a moderate deterioration in the baseline renal function was noted, with increased levels of serum creatinine and blood urea nitrogen, suggesting a pre-renal origin of AKI. There was a rise in acute phase proteins and international normalized ratio (INR) was prolonged. Serum electrolytes and blood levels of transaminases, alkaline phosphatase, bilirubin, lipase, and complete blood count were normal (Table 1). Urine dipstick was positive for leukocytes, erythrocytes, and protein. 
Table 1 Laboratory data upon arrival and at discharge from hospital

\begin{tabular}{|c|c|c|c|}
\hline Variable & Reference range, adults & On arrival & At discharge \\
\hline Hemoglobin (g/dl) & $13.5-17.5$ & 12.3 & 11.1 \\
\hline Hematocrit (\%) & $41.0-53.0$ & 37 & 33.5 \\
\hline Mean corpuscular volume (fl) & $80.0-94.0$ & 92.5 & 92.5 \\
\hline White-cell count (per $\mu \mathrm{l})$ & $4800-10,800$ & 8690 & 5900 \\
\hline \multicolumn{4}{|l|}{ Differential count (\%) } \\
\hline Neutrophils & $40.0-70.0$ & 53.2 & 66.7 \\
\hline Lymphocytes & $22.0-44.0$ & 2.8 & 19.5 \\
\hline Monocytes & $4.0-11.0$ & 12.0 & 9.7 \\
\hline Eosinophils & $0.0-8.0$ & 2.1 & 3.6 \\
\hline Platelet count (per $\mu \mathrm{l})$ & $130,000-400,000$ & 205,000 & 194,000 \\
\hline Sodium $(\mathrm{mmol} / \mathrm{l})$ & $135-145$ & 145 & 139 \\
\hline Potassium (mmol/l) & $3.3-5.1$ & 4.8 & 4.6 \\
\hline Chloride (mmol/l) & $96-108$ & 108 & \\
\hline Calcium (mg/dl) & $8.40-10.20$ & 8.70 & \\
\hline Magnesium (mg/dl) & $1.73-2.26$ & 2.24 & \\
\hline Urea nitrogen $(\mathrm{mg} / \mathrm{dl})$ & $15-50$ & 121 & 57 \\
\hline Creatinine (mg/dl) & $0.72-1.18$ & 3.18 & 1.81 \\
\hline Lactate $(\mathrm{mmol} / \mathrm{l})$ & $0.5-2.0$ & 1.1 & \\
\hline Glucose (mg/dl) & $70-110$ & 102 & \\
\hline Alanine aminotransferase (IU/l) & $9-59$ & 48 & \\
\hline Aspartate aminotransferase (IU/l) & $10-35$ & 93 & \\
\hline$\gamma$-glutamiltransferase (IU/l) & $8-61$ & 37 & \\
\hline Alkaline phosphatase (IU/l) & $40-129$ & 73 & \\
\hline Bilirubin (total) (mg/dl) & $0.12-1.10$ & 0.39 & \\
\hline Lipase (UI/l) & $13.0-60.0$ & 45 & \\
\hline Albumin (g/dl) & $3.4-4.8$ & 3.4 & \\
\hline Creatine kinase (IU/l) & $38-174$ & 203 & \\
\hline Lactate dehydrogenase (IU/l) & $135-225$ & 335 & \\
\hline C-reactive protein $(\mathrm{mg} / \mathrm{dl})$ & $<0.5$ & 8.77 & \\
\hline International normalized ratio & $0.84-1.20$ & 1.72 & \\
\hline APTT ratio & $0.86-1.20$ & 1.01 & \\
\hline Folate $(\mu \mathrm{g} / \mathrm{l})$ & $4.6-18.7$ & 2.5 & \\
\hline Vitamin B12 (ng/l) & $191-663$ & 384 & \\
\hline Serum iron $(\mu \mathrm{g} / \mathrm{dl})$ & $59-158$ & 63 & \\
\hline Serum transferrin $(\mathrm{mg} / \mathrm{dl})$ & $200-360$ & 190 & \\
\hline Ferritin $(\mu \mathrm{g} / \mathrm{l})$ & $30-400$ & 275 & \\
\hline \multicolumn{4}{|l|}{ Peripheral venous blood gases } \\
\hline $\mathrm{pH}$ & $7.32-7.42$ & 7.32 & \\
\hline Bicarbonate (mEq/l) & $20-24$ & 17.5 & \\
\hline
\end{tabular}

Reference ranges used at the IRCCS Ca' Granda Ospedale Maggiore Policlinico (Milano) are for adults who are not pregnant and do not have medical conditions that could affect the result. They may therefore not be appropriate for all patients
Abdominal radiography confirmed the known nephrolithiasis but did not show any remarkable pathological sign. Chest radiography and computed tomography (CT) of the thorax were compared to those acquired ten months before during pneumological follow-up and showed an evolution of the known IPF. Nasopharyngeal swab for SARS-Cov-2 tested negative. Blood, urine, and faeces were obtained for culture, and the patient was started on empirical antimicrobial therapy with Ceftriaxone and volume support with Lactated Ringer's solution.

The patient was admitted to the internal medicine unit for further medical testing and treatment. The gastrointestinal symptoms improved during the first days of hospitalization, as well as renal function. Stool Clostridium difficile toxin 
test, urine and blood cultures tested negative, while stool culture tested positive for Campylobacter jejuni.

During hospitalization, the patient reported frequent presyncopal symptoms. The episodes were characterized by blurry vision, diaphoresis, light-headedness, and in some cases palpitations and chest discomfort. They were mainly triggered by orthostatism, although in some cases could occur in the supine or sitting position, and they were not associated with specific situational triggers. No complete T-LOCs were documented during the hospital stay, but despite the correction of volume depletion and diarrhoea, the patient kept on experiencing presyncope.

\section{Further investigation and differential diagnosis}

Dr. Bozzano: continuous telemetric monitoring and serial ECGs showed occasional brief runs of rapid paroxysmal supraventricular tachycardia (PSVT) with spontaneous regression. Morphology was compatible with atrioventricular nodal re-entrant tachycardia, and there was no close correlation with the occurrence of presyncopal symptoms. Pacemaker was tested and it was normally functioning in DDD mode; the recordings showed occasional self-limited tachyarrhythmias with similar PSVT pattern.

Transthoracic echocardiography (TTE) revealed a normal cardiac pump function, with preserved left ventricular outflow tract gradients and no signs of aortic stenosis or hypertrophic cardiomyopathy.

Dr. Montinaro: once potentially high-risk cardiac disease had been ruled out, patient was examined for other causes of syncope. Orthostatic challenge showed a progressive and sustained BP fall from 140/70 to $80 / 40 \mathrm{mmHg}$ changing from the supine to the upright position within 3 min of active standing. HR increased from 61 to $71 \mathrm{bpm}$ during the challenge. The fall of BP was able to evoke the presyncopal symptoms, thus defining a classical orthostatic hypotension $(\mathrm{OH})$.

Prof. Tobaldini: all antihypertensive agents taken chronically by the patient were stopped, such as the alpha blocker silodosin and beta-blocker bisoprolol, and anticoagulation was interrupted due to the risk of fall. Symptoms were not affected by therapy discontinuation; therefore, further work up was performed due to the high suspicion of primary or secondary autonomic dysfunction.

Dr. Carandina: head-up tilt-table testing (TTT) was performed and confirmed a severe $\mathrm{OH}$ with associated chronotropic incompetence (Fig. 1). The absence of BP overshoot and of HR increase during the Valsalva manoeuvre were particularly indicative of a neurogenic $\mathrm{OH}$ due to autonomic failure. Twenty-four-hour ambulatory blood pressure monitoring (ABPM) revealed a nocturnal "non-dipping" BP

\begin{tabular}{|c|c|c|c|c|}
\hline EVENTS & $\begin{array}{c}\text { Time } \\
(\mathrm{min})\end{array}$ & $\begin{array}{c}\text { Systolic BP } \\
(\mathrm{mmHg})-\end{array}$ & $\begin{array}{c}\text { Diastolic BP } \\
(\mathrm{mmHg})-\end{array}$ & $\begin{array}{c}\text { HR } \\
(\mathrm{bpm})-\mathbf{L}\end{array}$ \\
\hline Baseline & 5 & 150 & 80 & 60 \\
\hline Tilt 75 & 10 & 115 & 70 & 63 \\
\hline Tilt 75 & 15 & 120 & 60 & 60 \\
\hline Tilt 75 & 20 & 90 & 60 & 60 \\
\hline Tilt 75 & 25 & 90 & 50 & 59 \\
\hline Recovery & 26 & 90 & 50 & 65 \\
\hline Recovery & 30 & 100 & 50 & 63 \\
\hline
\end{tabular}

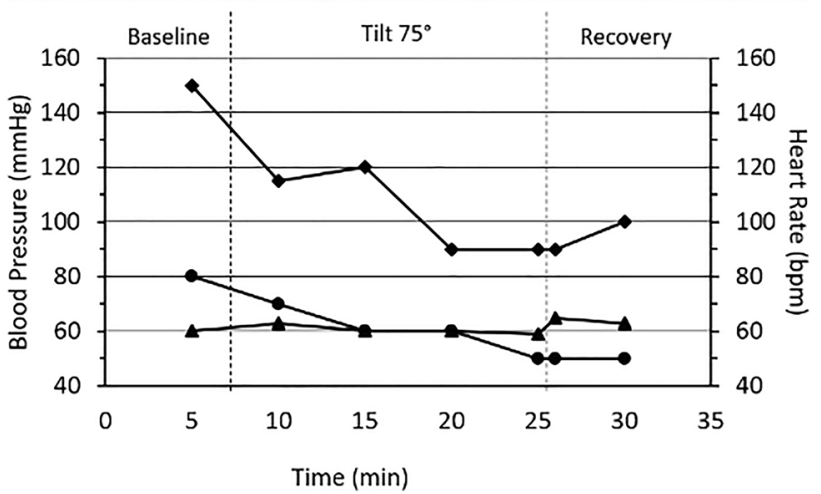

Fig. 1 Head-up tilt table test results: blood pressure (BP) and heart rate $(\mathrm{HR})$ trends during the phases of tilt test $\left(75^{\circ}\right)$

pattern, which furtherly supported the diagnosis of autonomic failure.

Dr. Di Fonzo: neurological examination and clinical history were not suggestive of neurodegenerative diseases such as Parkinson's disease, dementia with Lewy bodies, pure autonomic failure or multiple system atrophy, that may have autonomic failure as part of the clinical picture. Electromyography (EMG) showed a normal nerve conduction and the absence of peripheral neuropathies. CT scan of the brain was normal.

Prof. Tobaldini: causes of secondary autonomic failure were searched. Autonomic neuropathy involves postganglionic autonomic nerves and is most commonly caused by diabetes, or uremia, even though $\mathrm{OH}$ is rarely the presenting feature. Alcohol consumption has a strong association with dysautonomia too and should always be excluded prior to proceed in differential diagnosis. Medical history, patient's habits and labs were reviewed and were unconvincing for alcohol-related, uremic, and diabetic aetiologies (Table 1). Other relatively frequent causes of secondary autonomic failure include paraneoplastic neuropathy-which occurs commonly in association with anti-Hu antibodies in patients with small cell lung cancer-autoimmune conditions such as Sjögren syndrome and other collagen vascular disorders, amyloidosis, spinal cord injuries, B12 deficiency, infections (e.g. syphilis, Lyme disease, HIV, Chagas), sarcoidosis, porphyria, and congenital dysautonomia (e.g. Riley-Day syndrome). Laboratory, familial history and clinical findings were inconsistent with the majority of the conditions listed above, while systemic amyloidosis remained a still 
viable diagnostic hypothesis. Therefore, decision was made to request a fat pad biopsy.

Dr. Croci: abdominal fat pad biopsy was carried out at periumbilical site by the plastic surgery service and specimen was processed with Congo red staining and examined using polarizing microscopy. Microscopy was positive for green birefringence under polarized light, compatible with systemic amyloidosis (Fig. 2).

Dr. Montinaro: immunohistochemistry was not available in our hospital to identify the type of protein subunit forming fibrils in our patient. Light chain amyloidosis (AL amyloidosis) was reasonably ruled out by excluding an underlying plasma cell proliferative disease - such as multiple myeloma, smoldering myeloma, solitary plasmacytoma or Waldenström macroglobulinemia—as hemoglobin and serum calcium levels were normal, no monoclonal protein was identified at serum protein electrophoresis, Bence-Jones urine protein test was negative and although serum free light chains (FLC) were increased, a normal ratio kappa/lambda was found (Table 2).

Dr. Bozzano: patient was discharged and referred to a highly specialized centre for management and follow-up of systemic amyloidosis. The syncopal episodes were to refer to a reduction in peripheral resistances, due to autonomic failure secondary to amyloidosis.

\section{Discussion}

Prof. Tobaldini: $\mathrm{OH}$ is a very rare cause of syncope in population $<65$ years [1], but its relevance exponentially increases when considering elderly patients $(30 \%$ of $>75$ years with

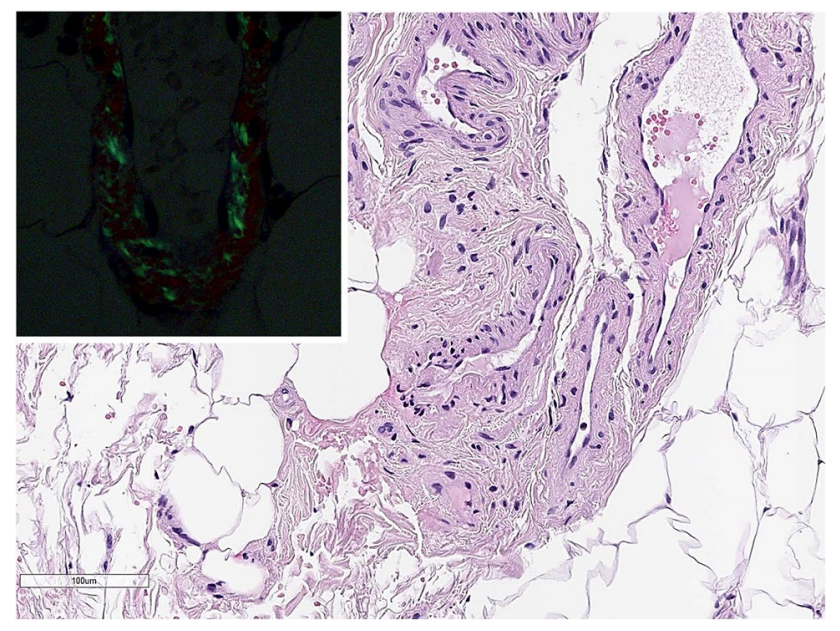

Fig. 2 Histologic examination (Hematoxylin/Eosin, 100×) displays an unremarkable subcutaneous adipose tissue, but featuring the presence of small vessels with subtle thickening of the walls. Congo red stain (inset) showed green birefringence under polarized light, consistent with the clinical suspicion of amyloidosis
Table 2 Serum protein electrophoresis, serum free light chains and urinary protein electrophoresis

\begin{tabular}{lll}
\hline Variable & Reference range, adults & Measured \\
\hline Serum protein electrophoresis & & \\
A/G ratio & $1.30-2.10$ & 1.14 \\
Albumin (\%) & $55.8-66.1$ & 53.2 \\
Alpha-1 globulins (\%) & $2.9-4.9$ & 5.7 \\
Alpha-2 globulins (\%) & $7.1-11.8$ & 10.9 \\
Beta-1 globulins (\%) & $4.7-7.2$ & 6.7 \\
Beta-2 globulins (\%) & $3.2-6.5$ & 7.8 \\
Gamma globulins (\%) & $11.1-18.8$ & 15.7 \\
Serum total protein (g/dl) & $6.40-8.00$ & 6.30 \\
Serum free light chains & & \\
Chain kappa (mg/liter) & $3.30-19.40$ & 45.05 \\
Chain lambda (mg/liter) & $5.71-26.30$ & 27.52 \\
Kappa/lambda ratio & $0.26-1.65$ & 1.63 \\
Urinary light chains (Bence & Absent & Absent \\
Jones protein) & & \\
\hline
\end{tabular}

Reference ranges used at the IRCCS $\mathrm{Ca}^{\prime}$ Granda Ospedale Maggiore Policlinico (Milano) are for adults who are not pregnant and do not have medical conditions that could affect the result. They may therefore not be appropriate for all patients

syncope [2]). As seen in our patient, reaching a final diagnosis can be challenging because of the presence of multiple confounding factors that could trigger or mimic syncope, including comorbidities and chronic therapy. When $\mathrm{OH}$ is suspected or documented at orthostatic challenge-defined in its "classical" form as a sustained decrease in systolic $\mathrm{BP} \geq 20 \mathrm{mmHg}$, diastolic $\mathrm{BP} \geq 10 \mathrm{mmHg}$, or a sustained decrease in systolic BP to an absolute value $<90 \mathrm{mmHg}$ within 3 min of active standing [3] - the first approach should include discontinuation of potentially aggravating medications and restoration of a normal plasmatic volume prior to further diagnostic evaluation. If symptoms persist, primary or secondary impairment of autonomous nervous system should be taken into account [4].

Dr. Di Fonzo: neurological evaluation to rule out neurodegenerative forms and peripheral neuropathies, EMG, head-up TTT, 24-h ABPM are useful tools to perform differential diagnosis among primary and secondary forms of dysautonomia but require a dedicated setting and should be performed by a specialist trained in autonomic function testing and interpretation [5].

Dr. Montinaro: neurogenic orthostatic hypotension $(\mathrm{nOH})$ can be common in systemic amyloidosis, especially in hereditary transthyretin amyloidosis (h-ATTR) which is the most commonly associated subtype [7]. Amyloidosis is a group of rare protein misfolding disorders, in which insoluble fibrillar proteins, or amyloids, deposit extracellularly and cause end-organ damage. Depending on the precursor protein, clinical manifestations in amyloidosis vary significantly. 
Extracellular accumulation of abnormally misfolded transthyretin (TTR) leads to ATTR, a multisystemic disorder whose clinical spectrum include sensory-motor neuropathy, cardiac, ocular and less commonly renal involvement [6]. Wild type TTR amyloidosis (wt-ATTR) is typically senile and manifests with a prominent cardiac involvement, while autonomic disfunction is a hallmark of h-ATTR amyloidosis and can include orthostatic hypotension, gastrointestinal dysmotility, erectile disfunction, urinary incontinence, and xerostomia [7]. In a recently published review, it is stated that $\mathrm{nOH}$ is a prominent and disabling manifestation of autonomic dysfunction in patients with h-ATTR amyloidosis, affecting an estimated $40-60 \%$ of patients and severely impacting their quality of life [8]. Existing links between systemic amyloidosis and autonomic dysfunction are known since long time, although pathophysiological mechanism is still not fully understood.

Dr Croci: in our patient, the diagnosis of systemic amyloidosis was made through the biopsy of periumbilical subcutaneous fat. It is an easy, low-cost and low-risk intervention, with a diagnostic sensitivity of $57-85 \%$, which is higher in patients with systemic amyloidosis due to immunoglobulin light chain (primary, or AL), AA protein (secondary), or TTR (senile cardiac or familial amyloid polyneuropathy) deposition, and it is lower in in patients with a single organ involved [9].

Dr. Montinaro: history taking, laboratory findings, urine analyses and FLC assay were used to rule out primary AL ad secondary AA amyloidosis in our patient. Based on peculiar presentation of the disease with $\mathrm{nOH}$ alone and no cardiac hypertrophic involvement, h-ATTR amyloidosis was the most likely diagnosis.

Dr. Bozzano: getting to a final diagnosis of amyloidosis in patients presenting with $\mathrm{nOH}$ alone can be challenging but might significantly improve prognosis and quality of life, since $\mathrm{nOH}$ can be treated when it is recognized [8]. Discontinuation of potentially aggravating medications, patient education and non-pharmacologic approaches should be applied first [7]. Droxidopa, a synthetic norepinephrine precursor, has shown efficacy in controlled trials of $\mathrm{nOH}[10]$ and is now approved in the US and Asia for patients with h-ATTR amyloidosis [8]. A number of novel drugs directed against amyloid precursor production (e.g. Patisiran, Inotersen) and fibril formation, or promoting TTR tetrameters stabilization (e.g. Tafamidis, Diflunisal) are being investigated and have shown promising results in randomized controlled trials by improving quality-of-life [11-14].

Prof. Tobaldini: finally, in elderly patients with unexplained $\mathrm{OH}$ and syncope, it is important to carry out a complete differential diagnosis. This includes ruling out cardiac etiology and major confounding factors, such as iatrogenic hypotension and hypovolemia. It is fundamental to address primary neurodegenerative dysautonomia and other common causes of dysautonomia, such as alcohol consumption and uremia. Been all the above excluded, amyloidosis should always be suspected and investigated.

Author contributions $\mathrm{BM}$ and ET contributed to the ideation and drafting of the manuscript. VB, AC, GAC, ADF and ET contributed to revision of the manuscript.

\section{Declarations}

Conflict of interest The authors declare that they have no conflict of interest related to the publication of the manuscript.

Statements on human and animal rights This article does not contain any studies with human participants or animals performed by any of the authors.

Informed consent For this type of study, formal consent is not required.

\section{References}

1. Del Rosso A, Alboni P, Brignole M, Menozzi C, Raviele A (2005) Relation of clinical presentation of syncope to the age of patients. Am J Cardiol 96(10):1431-1435. https://doi.org/10. 1016/j.amjcard.2005.07.047

2. Ungar A, Mussi C, Del Rosso A, Noro G, Abete P, Ghirelli L, Cellai T, Landi A, Salvioli G, Rengo F, Marchionni N, Masotti G (2006) Diagnosis and characteristics of syncope in older patients referred to geriatric departments. J Am Geriatr Soc 54(10):1531-1536. https://doi.org/10.1111/j.1532-5415.2006. 00891.x

3. Shibao C, Lipsitz LA, Biaggioni I (2013) ASH position paper: evaluation and treatment of orthostatic hypotension. J Clin Hypertens (Greenwich) 15:147-153. https://doi.org/10.1111/jch.12062

4. Brignole $\mathrm{M}$ et al (2018) 2018 ESC guidelines for the diagnosis and management of syncope (supplementary data). Eur Heart $\mathbf{J}$ 00:1-12. https://doi.org/10.1093/eurheartj/ehy037

5. Brignole $\mathrm{M}$ et al (2018) 2018 ESC guidelines for the diagnosis and management of syncope. Eur Heart J 39(21):1883-1948. https://doi.org/10.1093/eurheartj/ehy037

6. Girnius S (2013) Overview of systemic and localized amyloidosis. Rev Health Care 4(4):231-247. https://doi.org/10.7175/ rhc.v $4 \mathrm{i} 4.662$

7. Gonzalez-Duarte A, Valdés-Ferrer S, Cantù-Brito C (2019) Characteristics and natural history of autonomic involvement in hereditary ATTR amyloidosis : a systematic review. Clin Auton Res 29:1-9. https://doi.org/10.1007/s10286-019-00630-y

8. Palma JA, Gonzalez-Duarte A, Kaufmann H (2019) Orthostatic hypotension in hereditary transthyretin amyloidosis: epidemiology, diagnosis and management. Clin Auton Res 29(Suppl 1):33-44. https://doi.org/10.1007/s10286-019-00623-x

9. Westermark P (2012) Subcutaneous adipose tissue biopsy for amyloid protein studies. Methods Mol Biol 849:363-371. https://doi.org/10.1007/978-1-61779-551-0_24

10. Kaufmann H, Freeman R, Biaggioni I, Low P, Pedder S, Hewitt LA, Mauney J, Feirtag M, Mathias CJ (2014) Droxidopa for neurogenic orthostatic hypotension: a randomized, placebocontrolled, phase 3 trial. Neurology 83(4):328-335. https://doi. org/10.1212/WNL.0000000000000615 
11. Adams D et al (2018) Patisiran, an RNAi therapeutic, for hereditary transthyretin amyloidosis. N Engl J Med 379(1):11-21. https://doi.org/10.1056/NEJMoa1716153

12. Benson MD et al (2018) Inotersen treatment for patients with hereditary transthyretin amyloidosis. N Engl J Med 379(1):22-31. https://doi.org/10.1056/NEJMoa1716793

13. Coelho T et al (2012) Tafamidis for transthyretin familial amyloid polyneuropathy: a randomized, controlled trial. Neurology 79(8):785-792. https://doi.org/10.1212/WNL.0b013e3182661eb1
14. Berk JL et al (2013) Repurposing diflunisal for familial amyloid polyneuropathy: a randomized clinical trial. JAMA 310(24):26582667. https://doi.org/10.1001/jama.2013.283815

Publisher's Note Springer Nature remains neutral with regard to jurisdictional claims in published maps and institutional affiliations. 